\title{
The impact of helmet use on neurosurgical care and outcomes after pediatric all-terrain vehicle and dirt bike crashes: a 10-year single-center experience
}

\author{
*Jackson H. Allen, BA, ${ }^{1}$ Aaron M. Yengo-Kahn, MD, ${ }^{2}$ Kelly L. Vittetoe, BS, ${ }^{1}$ \\ Amber Greeno, MSN, APRN, ${ }^{3}$ Muhammad Owais Abdul Ghani, MBBS, ${ }^{3}$ Purnima Unni, MPH, ${ }^{3}$ \\ Harold N. Lovvorn III, MD, ${ }^{3}$ and Christopher M. Bonfield, MD² \\ ${ }^{1}$ Vanderbilt University School of Medicine, Nashville; and Departments of ${ }^{2}$ Neurological Surgery and ${ }^{3}$ Pediatric Surgery, \\ Vanderbilt University Medical Center, Nashville, Tennessee
}

\begin{abstract}
OBJECTIVE All-terrain vehicle (ATV) and dirt bike crashes frequently result in traumatic brain injury. The authors performed a retrospective study to evaluate the role of helmets in the neurosurgical outcomes of pediatric patients involved in ATV and dirt bike crashes who were treated at their institution during the last decade.
\end{abstract}

METHODS The authors analyzed data on all pediatric patients involved in ATV or dirt bike crashes who were evaluated at a single regional level I pediatric trauma center between 2010 and 2019. Patients were excluded if the crash occurred in a competition $(n=70)$ or if helmet status could not be determined $(n=18)$. Multivariable logistic regression was used to analyze the association of helmet status with the primary outcomes of 1) neurosurgical consultation, 2) intracranial injury (including skull fracture), and 3) moderate or severe traumatic brain injury (MSTBI) and to control for literaturebased, potentially confounding variables.

RESULTS In total, 680 patients were included (230 [34\%] helmeted patients and 450 [66\%] unhelmeted patients). Helmeted patients were more frequently male $(81 \%$ vs $66 \%)$. Drivers were more frequently helmeted $(44.3 \%)$ than passengers $(10.5 \%, p<0.001)$. Head imaging was performed to evaluate $70.9 \%$ of unhelmeted patients and $48.3 \%$ of helmeted patients $(p<0.001)$. MSTBI $(8.0 \%$ vs $1.7 \%, p=0.001)$ and neurosurgical consultation $(26.2 \%$ vs $9.1 \%, p<0.001)$ were more frequent among unhelmeted patients. Neurosurgical injuries, including intracranial hemorrhage $(16 \%$ vs $4 \%, p<$ $0.001)$ and skull fracture (18\% vs $4 \%, p<0.001)$, were more common in unhelmeted patients. Neurosurgical procedures were required by $2.7 \%$ of unhelmeted patients. One helmeted patient $(0.4 \%)$ required placement of an intracranial pressure monitor, and no other helmeted patients required neurosurgical procedures. After adjustment for age, sex, driver status, vehicle type, and injury mechanism, helmet use significantly reduced the odds of neurosurgical consultation (OR $0.250,95 \% \mathrm{Cl} 0.140-0.447, \mathrm{p}<0.001$ ), intracranial injury (OR 0.172, 95\% Cl 0.087-0.337, p < 0.001), and MSTBI (OR $0.244,95 \% \mathrm{Cl} 0.079-0.758, p=0.015)$. The unadjusted absolute risk reduction provided by helmet use equated to a number-needed-to-helmet of 6 riders to prevent 1 neurosurgical consultation, 4 riders to prevent 1 intracranial injury, and 16 riders to prevent $1 \mathrm{MSTBI}$.

CONCLUSIONS Helmet use remains problematically low among young ATV and dirt bike riders, especially passengers. Expanding helmet use among these children could significantly reduce the rates of intracranial injury and MSTBI, as well as the subsequent need for neurosurgical procedures. Promoting helmet use among recreational ATV and dirt bike riders must remain a priority for neurosurgeons, public health officials, and injury prevention professionals.

https://thejns.org/doi/abs/10.3171/2021.6.PEDS21225

KEYWORDS all-terrain vehicle; ATV; dirt bike; helmet; traumatic brain injury; injury prevention; public health; trauma

ABBREVIATIONS ATV = all-terrain vehicle; ED = emergency department; EVD = extraventricular drain; GCS = Glasgow Coma Scale; GOS = Glasgow Outcome Scale; $I C P m=$ intracranial pressure monitor; ISS = Injury Severity Score; MSTBI = moderate or severe traumatic brain injury; TBI = traumatic brain injury; VPS = ventriculoperitoneal shunt.

SUBMITTED April 29, 2021. ACCEPTED June 7, 2021.

INCLUDE WHEN CITING Published online October 12, 2021; DOI: 10.3171/2021.6.PEDS21225.

* J.H.A. and A.M.Y.K. contributed equally to this work and share first authorship. 
$\mathrm{P}$ EDIATRIC injuries due to the use of all-terrain vehicles (ATVs) and dirt bikes continue to be a significant public health concern despite efforts to curb injuries among riders younger than 16 years..$^{1-7}$ The United States Consumer Product Safety Commission's most recent report on off-road vehicle crashes identified an average of 104,900 injuries per year from 2015 to 2019.8 Children younger than 16 years accounted for $27 \%$ of injuries during this period, and more than half of pediatric injuries occurred in children younger than 12 years. Children younger than 16 years also represented $16 \%$ of ATVrelated fatalities from 2015 to 2019, a disproportionately high rate relative to their representation in the population. ${ }^{8}$ In comparison with children involved in motor vehicle accidents, children injured while riding ATVs are twice as likely to require hospitalization. ${ }^{9}$ Pediatric injuries and fatalities related to ATV use are also associated with significant economic burden. ${ }^{10-13}$

Despite the passage of laws aimed at curbing pediatric injuries, risky behaviors such as riding without a helmet remain chronic problems among ATV riders, especially children. The vast majority of pediatric riders report engaging in at least one or more risky behaviors while on an ATV, such as riding with passengers $(92 \%)$, riding on public roads $(81 \%)$, or riding without a helmet $(64 \%) .{ }^{14}$ Other notable risk factors for pediatric injury include driver age, sex, experience, recreational use, driving patterns, vehicle characteristics, and crash mechanism..$^{5,6,15-18}$

Although similar in mechanism to injuries due to ATV crashes, pediatric dirt bike injuries are relatively poorly described in the recent literature, especially outside of competitive motocross events where helmet use is nearly universal. ${ }^{19-21}$ The risk factors are similar for these young riders, and a few single-center studies have demonstrated a preponderance of extremity and head injuries among this group..$^{22,23}$

Head injuries consistently comprise a substantial proportion of all ATV-related injuries, including $27 \%$ of such injuries in 2016. ${ }^{8}$ ATV-related head injuries may occur as frequently as extremity fractures in the pediatric population. ${ }^{8,19}$ Although multiple single-center studies have highlighted head injury as a source of morbidity and mortality, there remains a lack of detail in the reporting of specific head injuries that require neurosurgical consultation or intervention, such as intracranial hemorrhage, skull fracture, and moderate or severe traumatic brain injury (MSTBI) ${ }^{24-27}$ Furthermore, although helmet use has been shown to reduce head injury in other settings, such as motorcycle and bicycle crashes, there are limited data on the role of helmets in preventing specific intracranial injuries or the need for emergency neurosurgical interventions for patients injured on ATVs or recreational dirt bikes. ${ }^{28-30}$ Despite substantial changes in recreational vehicle use and the adoption of legislation regarding ATV use in multiple states, data on ATV crashes and head injuries in the pediatric population have been infrequently updated over the last 2 decades. ${ }^{25}$

Head injury represents a particularly high-acuity subset of all pediatric ATV and dirt bike-related injuries, and consistently updated reporting is important to inform public health initiatives. Recognizing these issues, we per- formed a retrospective cohort study to better characterize the spectrum of neurosurgical injuries related to ATV and dirt bike crashes and to describe the effectiveness of helmet use in preventing these injuries over a recent 10-year period. We hypothesized that helmet use during ATV and dirt bike crashes would be associated with decreased rates of intracranial injuries and reduced need for neurosurgical intervention.

\section{Methods \\ Study Design}

The data analyzed for this retrospective cohort study were obtained from a prospectively collected pediatric trauma registry that contained records for all patients who presented with traumatic injuries to a large level I pediatric trauma center in the southeastern United States between January 1, 2010, and December 31, 2019. The study was approved by our institutional review board, and data accession and storage were performed in accordance with the Health Insurance Portability and Accountability Act.

\section{Study Population}

The included patients were evaluated by the pediatric trauma surgery service after an ATV or dirt bike crash during the study period. Of note, our institutional standard of practice is for patients who are older than 16 years and involved in an ATV or dirt bike crash to be sent to the adult hospital for treatment, but no strict age-based exclusion criteria were applied for the purpose of this study. Patients were excluded if the crash occurred in a competition event $(\mathrm{n}=70)$ or if helmet status could not be determined $(n=18)$.

\section{Variables}

Patient records were manually reviewed, and the extracted data were stored securely using REDCap (Research Electronic Data Capture). ${ }^{31}$ Key injury variables collected from the patient records included demographic characteristics, mechanism of injury (rollover crash, crash during jump, ejection, collision with solid structure, collision with another vehicle), location of crash (home/yard, street, farm/field, or racetrack), driver status (passenger or driver), vehicle type (ATV or dirt bike), intubation prior to hospital arrival, and Glasgow Coma Scale (GCS) score and Injury Severity Score (ISS) on admission. Helmet status was defined as helmeted or unhelmeted on the basis of first responder documentation, as well as initial clinical notes that documented the patient's presentation.

The collected neurosurgical variables included the results of any head or brain imaging studies performed, including the presence and type of skull fracture (basilar, calvarial, depressed, or open). Intracranial hemorrhage was categorized as single or mixed, and further classified according to nonmutually exclusive patterns (epidural, subdural, subarachnoid, intraparenchymal, and/or intraventricular). Radiographic variables were included in reports by board-certified radiologists, and imaging studies were manually reviewed by the senior author (C.M.B.) when reports were unavailable. The occurrence of a neurosurgical consultation was defined by the presence of a 
consultation note. Neurosurgical procedures were categorized on the basis of the operative and procedure notes. The decision to pursue neurosurgical intervention was at the discretion of the attending neurosurgeon.

\section{Outcomes}

Primary outcomes included 1) neurosurgical consultation, 2) intracranial injury (skull fracture and/or intracranial hemorrhage), 3) neurosurgical procedure, 4) MSTBI (GCS scores 3-12 at presentation), and 5) length of hospitalization (days). Secondary outcomes included functional status at last follow-up, as defined using the Glasgow Outcome Scale (GOS) in which scores range $1-5$, in which 1 corresponds to death and 5 corresponds to a normal life with minor neurological deficits. ${ }^{32}$ In accordance with a previously reported study, a "good functional outcome" was defined as GOS score 4 or 5 at the last follow-up visit. ${ }^{33}$

\section{Statistical Analysis}

Categorical variables were presented as frequency and proportion. Continuous variables were presented as mean $\pm \mathrm{SD}$, except those with nonnormal distributions were presented as median (interquartile range). Observed outcomes were stratified according to helmet status. Chisquare analysis was used to compare categorical variables, and the independent-samples t-test or Mann-Whitney Utest was used to compare continuous variables depending on the nature of their distribution.

Covariates were selected a priori for inclusion in the multivariable analysis on the basis of their hypothetical potential for interactions between demographic characteristics (e.g., age, sex, and driver status) and helmet status and/or possible confounding with outcomes (e.g., severe mechanism of injury). ${ }^{5,6,14-18}$ The one-in-ten rule was used to limit the inclusion of preselected independent variables in all regression analyses on the basis of the count of the rarest outcome. ${ }^{34,35}$ Single-step multivariable logistic regression was performed to consider the effects of helmet use, age, sex, driver status, vehicle type, and mechanism of crash on the occurrence of neurosurgical consultation. This approach was repeated for intracranial injury.

A third multivariable logistic regression was used to analyze the association between MSTBI (GCS scores 3-12) and helmet use, age, driver status, and vehicle type. In this analysis, the number of included independent variables was limited by the sample size. Analysis was performed with SPSS Statistics version 27 (IBM Corp.). Statistical significance was set a priori at $\mathrm{p}<0.05$.

\section{Results}

\section{Demographic and General Injury Characteristics}

A total of 680 patients met the inclusion criteria (mean [SD] age 11.4 [3.8] years, 71.3\% male, and 93.0\% White). Most riders $(66 \%)$ were unhelmeted when the crash occurred; the majority were riding ATVs (88.7\%) and driving $(58.2 \%)$. The majority of ATV riders were unhelmeted $(78.2 \%)$, whereas most dirt bike riders were helmeted $(70.0 \%)$. Male riders had a higher rate of helmet use than female riders $(38.6 \%$ vs $22.1 \%, \mathrm{p}<0.001)$. Drivers were more frequently helmeted $(44.3 \%)$ than passengers $(10.5 \%$, $\mathrm{p}<0.001)$. Mechanism and location of crash were similar between helmeted and unhelmeted riders. Unhelmeted riders were more frequently intubated prior to hospital arrival $(5.1 \%$ vs $1.3 \%, \mathrm{p}=0.014)$ and more frequently admitted to the ICU from the emergency department (ED) (19.8\% vs $9.6 \%$, p < 0.001). Full demographic, crash, and admission variables are presented in Table 1.

\section{Neurosurgical Injuries and Procedures}

Compared with helmeted riders, unhelmeted riders more frequently underwent head imaging $(70.9 \%$ vs $48.3 \%, \mathrm{p}<0.001$ ), received neurosurgical consultation ( $26.2 \%$ vs $9.1 \%, \mathrm{p}<0.001)$, and experienced MSTBI $(8.0 \%$ vs $1.7 \%, p=0.001)$. Unhelmeted riders more frequently experienced skull fracture (17.8\% vs $4.3 \%, \mathrm{p}<0.001)$ and intracranial hemorrhage $(16.2 \%$ vs $3.9 \%, \mathrm{p}<0.001)$. Neurosurgical procedures, such as extraventricular drain (EVD) placement, intracranial pressure monitor (ICPm) placement, ventriculoperitoneal shunt (VPS) placement, craniotomy, or craniectomy, were required by $2.7 \%$ of unhelmeted riders. One helmeted rider required ICPm placement; no helmeted riders required an EVD, craniotomy, craniectomy, or VPS. Three unhelmeted riders required 6, 4 , and 2 procedures, respectively. No helmeted riders required multiple procedures. The unadjusted absolute risk reduction provided by helmet use equated to a numberneeded-to-helmet of 6 riders to prevent 1 neurosurgical consultation, 4 riders to prevent 1 intracranial injury, and 16 riders to prevent 1 MSTBI. Further characterization of the neurosurgical injuries and procedures is shown in Table 2.

\section{Clinical Outcomes}

Length of hospitalization and final hospital disposition were similar between helmeted and unhelmeted riders. Median length of hospitalization was 2 days for both helmeted and unhelmeted riders $(\mathrm{U}=53,404, \mathrm{p}=0.48)$. Two helmeted riders died. One death was due to neurotrauma, and this rider underwent ICPm placement. The second death was related to cardiopulmonary arrest due to an extracranial injury. Three unhelmeted riders died, 2 of whom died of neurotrauma. GOS scores assessed at the most recent follow-up visits were similar between the groups, with $>98 \%$ of patients achieving a good clinical outcome (GOS score 4 or 5). Complete clinical outcomes are presented in Table 3.

\section{Multivariable Analysis}

After adjustment for age, sex, driver status, vehicle type, and injury mechanism, helmet use remained associated with significantly reduced odds of neurosurgical consultation (OR 0.25, 95\% CI 0.14-0.45, p < 0.001). Similarly, helmet use was independently associated with significantly reduced odds of intracranial injury (OR 0.17 , 95\% CI 0.09-0.38, p < 0.001). Helmeted riding was also associated with significantly reduced odds of MSTBI (OR $0.24,95 \%$ CI $0.08-0.76, p=0.015$ ) after adjustment for age, driver status, and vehicle type. The results of the full multivariable analysis are presented in Table 4. 
TABLE 1. Demographic characteristics, injury mechanisms, and injury severity

\begin{tabular}{|c|c|c|c|}
\hline Characteristic & $\begin{array}{l}\text { Helmet } \\
(n=230)\end{array}$ & $\begin{array}{l}\text { No Helmet } \\
(n=450)\end{array}$ & $\begin{array}{c}p \\
\text { Value }\end{array}$ \\
\hline \multicolumn{4}{|l|}{ Demographic } \\
\hline Age, yrs & $11.01 \pm 3.65$ & $11.69 \pm 3.89$ & 0.03 \\
\hline $1-5$ & $23(10)$ & $43(9.6)$ & 0.85 \\
\hline $6-11$ & $94(40.9)$ & $124(27.6)$ & $<0.001$ \\
\hline $12-17$ & $113(49.1)$ & $283(62.9)$ & $<0.001$ \\
\hline Male & 187 (81.3) & 298 (66.2) & $<0.001$ \\
\hline Race & & & 0.17 \\
\hline White & $220(95.7)$ & $413(91.8)$ & \\
\hline Black & $9(3.9)$ & $26(5.8)$ & \\
\hline Other & $1(0.4)$ & $5(1.1)$ & \\
\hline Unknown & $0(0.0)$ & $6(1.3)$ & \\
\hline Hispanic & $5(2.2)$ & $16(3.6)$ & \\
\hline \multicolumn{4}{|l|}{ Injury profile } \\
\hline Vehicle $^{*}$ & & & $<0.001$ \\
\hline ATV & $111(21.8)$ & $399(78.2)$ & \\
\hline Dirt bike & $119(70.0)$ & $51(30.0)$ & \\
\hline Driver status* & & & $<0.001$ \\
\hline Driver & $208(44.3)$ & $262(55.7)$ & \\
\hline Passenger & $22(10.5)$ & $188(89.5)$ & \\
\hline Mechanism of crash & & & $<0.001$ \\
\hline Rollover crash & $59(25.7)$ & $195(43.3)$ & \\
\hline Crash during jump & $15(6.5)$ & $6(1.3)$ & \\
\hline Ejected w/o collision & $60(26.1)$ & $116(25.8)$ & \\
\hline Collision w/ vehicle & $39(17.0)$ & $42(9.3)$ & \\
\hline Collision w/ object & $57(24.8)$ & $91(20.2)$ & \\
\hline Location & & & 0.15 \\
\hline Home/yard & $135(58.7)$ & $249(55.3)$ & \\
\hline Racetrack & $9(3.9)$ & $12(2.7)$ & \\
\hline Street & $52(22.6)$ & $87(19.3)$ & \\
\hline Farm/field & $27(11.7)$ & 85 (18.9) & \\
\hline Unspecified & $7(3.0)$ & $17(3.8)$ & \\
\hline Emergency transport & & & 0.004 \\
\hline Ground & $149(64.8)$ & $250(55.6)$ & \\
\hline Helicopter & $64(27.8)$ & $180(40.0)$ & \\
\hline Private & $17(7.4)$ & $20(4.4)$ & \\
\hline $\begin{array}{l}\text { Location before hospital } \\
\text { admission }\end{array}$ & & & 0.94 \\
\hline Scene & $84(36.5)$ & $161(35.8)$ & \\
\hline $\mathrm{OSH}$ & $144(62.6)$ & $286(63.6)$ & \\
\hline Urgent care/clinic & $2(0.9)$ & $3(0.7)$ & \\
\hline Intubated PTA & $3(1.3)$ & $23(5.1)$ & 0.014 \\
\hline ISS & & & 0.030 \\
\hline $1-8$ & $101(43.9)$ & $182(40.4)$ & \\
\hline $9-15$ & $98(42.6)$ & $162(36.0)$ & \\
\hline $16-24$ & $20(8.7)$ & $69(15.3)$ & \\
\hline $25-49$ & $11(4.8)$ & $34(7.6)$ & \\
\hline $50-75$ & $0(0.0)$ & $3(0.7)$ & \\
\hline
\end{tabular}

» CONTINUED FROM PREVIOUS COLUMN

TABLE 1. Demographic characteristics, injury mechanisms, and injury severity

OSH = outside hospital; PTA = prior to ED arrival.

Values are shown as mean \pm SD or number (percent) unless indicated otherwise.

* Percentages were calculated for each row (e.g., percentage of helmeted or unhelmeted riders among all ATV riders).

\section{Discussion}

This study aimed to characterize the impact of helmet use on head injuries and neurosurgical outcomes of ATV and dirt bike riders during the last decade. Pediatric patients continue to sustain serious head injuries related to unhelmeted riding of ATVs and dirt bikes. Specifically, unhelmeted riders more frequently presented with MSTBI, received head imaging, and were admitted to the ICU than helmeted riders. Furthermore, unhelmeted riders had 4 times the odds of neurosurgical consultation and nearly 6 times the odds of skull fracture or intracranial hemorrhage, independent of age, sex, driver status, vehicle type, and crash mechanism. Most importantly, in our cohort of patients treated between 2010 and 2019 only 1 dirt bike rider wearing a helmet required a neurosurgical procedure (ICPm placement). In this unfortunate circumstance, the helmeted rider received a devastating injury after being struck by a vehicle at highway speeds while riding on a public road; this was an atypical mechanism of injury. No helmeted riders required an EVD, VPS, craniotomy, or craniectomy during the study period.

Prior investigations of ATV and dirt bike crashes and related head injuries have yielded similar conclusions about the role of helmets in preventing ICU admission and general traumatic brain injury (TBI) ${ }^{28,36}$ The role of helmets in preventing specific intracranial injuries and the need for neurosurgical intervention has been less well characterized. For example, prior single-center studies have not studied or been sufficiently powered to characterize the relative risk of neurological injury in unhelmeted pediatric patients. . $^{15,18,24,25,28,37}$ Mangano et al. detected an increased, but statistically nonsignificant, rate of neurological injuries among unhelmeted riders in a cohort of 185 patients who were treated during the decade 1993-2003. ${ }^{25}$ Using National Trauma Data Bank records, Bowman et al. identified increased rates of TBI and death among unhelmeted ATV riders of all ages, but they did not specifically analyze data in pediatric patients or characterize the risks of specific neurosurgical injuries among unhelmeted riders..$^{28}$ Our analysis provides substantial granularity to the study of neurosurgical injuries among recreational vehicle riders and suggests that helmet use is critically important to reducing rates of intracranial injury and MSTBI, as well as subsequent neurosurgical procedures, in the pediatric population.

Although acute outcomes differed between helmeted and unhelmeted passengers, GOS scores were similar at follow-up. Because the vast majority of the injuries were mild TBIs ( $>90 \%$ ), the GOS is likely not sensitive enough to demonstrate important differences in neuropsychologi- 
TABLE 2. Neurosurgical injuries and treatments

\begin{tabular}{|c|c|c|c|}
\hline Characteristic & $\begin{array}{l}\text { Helmet } \\
(n=230)\end{array}$ & $\begin{array}{l}\text { No Helmet } \\
(n=450)\end{array}$ & $\begin{array}{c}p \\
\text { Value }\end{array}$ \\
\hline \multicolumn{4}{|l|}{ GCS score } \\
\hline $3-8$ & $4(1.7)$ & $26(5.8)$ & 0.015 \\
\hline $9-12$ & $0(0.0)$ & $10(2.2)$ & 0.019 \\
\hline $13-15$ & $226(98.3)$ & $414(92.0)$ & 0.001 \\
\hline Head imaging & $111(48.3)$ & $319(70.9)$ & $<0.001$ \\
\hline CT & $110(47.8)$ & $319(70.9)$ & $<0.001$ \\
\hline MRI & $7(3)$ & $10(2.2)$ & 0.52 \\
\hline CT angiography & $7(3)$ & $24(5.3)$ & 0.18 \\
\hline Skull fracture & $10(4.3)$ & $80(17.8)$ & $<0.001$ \\
\hline Basilar & $2(0.9)$ & $17(3.8)$ & 0.03 \\
\hline Calvarial & $8(3.5)$ & $63(14)$ & $<0.001$ \\
\hline Multiple & $6(2.6)$ & $37(8.2)$ & 0.004 \\
\hline Depressed & $1(0.4)$ & $15(3.3)$ & 0.018 \\
\hline Open & $0(0)$ & $2(0.4)$ & 0.55 \\
\hline Pneumocephalus & $7(3)$ & $41(9.1)$ & 0.003 \\
\hline Intracranial hemorrhage pattern & $9(3.9)$ & $73(16.2)$ & $<0.001$ \\
\hline $\mathrm{EDH}$ & $1(0.4)$ & $21(4.7)$ & 0.003 \\
\hline $\mathrm{SDH}$ & $3(1.3)$ & $24(5.3)$ & 0.01 \\
\hline $\mathrm{SAH}$ & $2(0.9)$ & $15(3.3)$ & 0.052 \\
\hline IVH & $0(0)$ & $4(0.9)$ & 0.31 \\
\hline IPH & $2(0.9)$ & $4(0.9)$ & 0.98 \\
\hline Mixed & $1(0.4)$ & $5(1.1)$ & 0.37 \\
\hline Neurosurgical consultation & $21(9.1)$ & $118(26.2)$ & $<0.001$ \\
\hline Neurosurgical procedure & $1(0.4)$ & $12(2.7)$ & 0.04 \\
\hline Multiple & $0(0)$ & $3(0.7)$ & 0.55 \\
\hline EVD placement & $0(0)$ & $4(0.9)$ & 0.31 \\
\hline ICPm placement & $1(0.4)$ & $6(1.3)$ & 0.27 \\
\hline Craniotomy & $0(0)$ & $7(1.6)$ & 0.10 \\
\hline Craniectomy & $0(0)$ & $2(0.4)$ & 0.55 \\
\hline VPS placement & $0(0)$ & $2(0.4)$ & 0.55 \\
\hline Cranioplasty & $0(0)$ & $4(0.9)$ & 0.31 \\
\hline
\end{tabular}

EDH = epidural hematoma; IPH = intraparenchymal hemorrhage; IVH = intraventricular hemorrhage; $\mathrm{SAH}=$ subarachnoid hemorrhage; $\mathrm{SDH}=$ subdural hematoma.

Values are shown as number (percent) unless indicated otherwise.

cal wellness between groups after injury. Although our large retrospective study relied on GOS scores, we must consider the subtle but critical benefits of helmets that may be reflected in academic achievement, delayed return to school, or persistent postconcussive symptoms. Detailed prospective analyses are needed to adequately evaluate these outcomes. According to the literature, MSTBI is associated with lower health-related quality of life, poorer social and emotional functioning, and increased neurobehavioral sequelae, even compared with mild TBI..$^{38,39}$ Thus, the increased rate of MSTBI among unhelmeted patients may be a proxy for these more subtle outcomes, despite relatively normal gross functional status as measured using the GOS. We consider any reduction in the risk of severe head injury to be helpful.
TABLE 3. Clinical outcomes

\begin{tabular}{|c|c|c|c|}
\hline Characteristic & $\begin{array}{l}\text { Helmet } \\
(n=230)\end{array}$ & $\begin{array}{l}\text { No Helmet } \\
(n=450)\end{array}$ & $\begin{array}{c}p \\
\text { Value }\end{array}$ \\
\hline \multicolumn{4}{|l|}{ ED disposition } \\
\hline Home & $21(9.1)$ & $39(8.7)$ & 0.84 \\
\hline Observation & $15(6.5)$ & $25(5.6)$ & 0.61 \\
\hline Floor & $140(60.9)$ & $243(54.0)$ & 0.087 \\
\hline ICU & $22(9.6)$ & $89(19.8)$ & $<0.001$ \\
\hline OR & $32(13.9)$ & $54(12.0)$ & 0.48 \\
\hline \multicolumn{4}{|l|}{ Hospital disposition } \\
\hline Home & $223(97.0)$ & $435(96.7)$ & 0.84 \\
\hline Home services/rehab & $4(1.7)$ & $5(1.1)$ & 0.48 \\
\hline DCPS & $1(0.4)$ & $1(0.2)$ & 0.63 \\
\hline Death (all-cause) & $2(0.9)$ & $3(0.7)$ & 0.77 \\
\hline Brain death & $1(0.4)$ & $2(0.4)$ & 0.99 \\
\hline Length of hospitalization, days & $2(1-3)$ & $2(1-3)$ & 0.48 \\
\hline No. in upper quartile & 37 (16.1) & $90(20.0)$ & 0.22 \\
\hline Days in ICU & $2.5 \pm 1.7$ & $3.6 \pm 6.8$ & 0.51 \\
\hline \multicolumn{4}{|l|}{ GOS score at follow-up } \\
\hline 1 (death) & $2(0.9)$ & $3(0.7)$ & 0.77 \\
\hline 2 (neurovegetative) & $0(0.0)$ & $0(0.0)$ & $>0.99$ \\
\hline 3 (severe disability) & $0(0.0)$ & $2(0.4)$ & 0.55 \\
\hline 4 (moderate disability) & $2(0.9)$ & $5(1.1)$ & 0.77 \\
\hline 5 (minor deficits) & $226(98.3)$ & $440(97.8)$ & 0.67 \\
\hline
\end{tabular}

DCPS = Department of Child Protective Services; OR = operating room.

Values are shown as number (percent), median (interquartile range), or mean \pm SD unless indicated otherwise.

Similarly, length of hospitalization was similar between these groups, but significant variability is attributed to extracranial injuries that require hospitalization and often accompany head injury. In the case of polytrauma experienced by many patients, it is unlikely that any reduction in head injuries afforded by helmet use is sufficient to reduce hospital stay when other injuries are present. Given that the median hospital stay was just 2 days for both groups, any increased length of stay may have been statistically undetectable owing to the more frequent and severe head injuries of the unhelmeted group.

Overall, the use of helmets may have relatively minor impacts on gross neurological function at follow-up or total length of hospitalization, but it does have a clear impact on intracranial injuries and TBI. The findings of the present study have important implications for the health and well-being of the substantial number of children who ride ATVs and dirt bikes every year. First, the rate of helmet use among this study population was relatively unchanged compared with those of prior analyses across geographic regions..$^{5,6,22,23,25,37}$ Expanding the use of helmets among all ATV and dirt bike riders could significantly reduce the likelihood and severity of head trauma for these children. As a result, promoting helmet use among recreational ATV and dirt bike riders must remain a priority for neurosurgeons, public health officials, and injury prevention professionals, and these findings pro- 
TABLE 4. Results of binary logistic regression analysis

\begin{tabular}{|c|c|c|c|c|c|c|}
\hline \multirow[b]{2}{*}{ Factor } & \multicolumn{2}{|c|}{ Neurosurgical Consultation } & \multicolumn{2}{|c|}{ Intracranial Injury } & \multicolumn{2}{|l|}{ MSTBI } \\
\hline & OR $(95 \% \mathrm{Cl})$ & $p$ Value & OR $(95 \% \mathrm{Cl})$ & $p$ Value & OR $(95 \% \mathrm{Cl})$ & p Value \\
\hline \multicolumn{7}{|l|}{ Helmet use } \\
\hline Yes & $0.250(0.140-0.447)$ & $<0.001$ & $0.172(0.087-0.337)$ & $<0.001$ & $0.244(0.079-0.758)$ & 0.015 \\
\hline No & Ref & & Ref & & Ref & \\
\hline $\mathrm{Age}^{*}$ & $1.064(1.005-1.125)$ & 0.032 & $1.019(0.96-1.082)$ & 0.532 & $1.062(0.966-1.168)$ & 0.212 \\
\hline \multicolumn{7}{|l|}{ Sex } \\
\hline Male & $0.736(0.479-1.132)$ & 0.163 & $0.891(0.554-1.433)$ & 0.633 & & \\
\hline Female & Ref & & Ref & & & \\
\hline \multicolumn{7}{|l|}{ Driver status } \\
\hline Driver & $1.186(0.749-1.879)$ & 0.467 & $1.160(0.703-1.915)$ & 0.562 & $0.939(0.486-1.913)$ & 0.863 \\
\hline Passenger & Ref & & Ref & & Ref & \\
\hline \multicolumn{7}{|l|}{ Vehicle } \\
\hline ATV & $0.763(0.431-1.352)$ & 0.354 & $0.634(0.345-1.163)$ & 0.141 & $1.364(0.486-3.828)$ & 0.555 \\
\hline Dirt bike & Ref & & Ref & & Ref & \\
\hline \multicolumn{7}{|l|}{ Mechanism of crash } \\
\hline Crash during jump & $1.518(0.454-5.074)$ & 0.498 & $1.270(0.266-6.062)$ & 0.764 & & \\
\hline Ejected w/o collision & $2.139(1.323-3.459)$ & 0.002 & $3.226(1.901-5.472)$ & $<0.001$ & & \\
\hline Collision w/ vehicle & $0.907(0.434-1.895)$ & 0.794 & $1.360(0.616-3.004)$ & 0.447 & & \\
\hline Collision w/ object & $0.635(0.348-1.158)$ & 0.139 & $0.950(0.491-1.836)$ & 0.878 & & \\
\hline Rollover crash & Ref & & Ref & & & \\
\hline
\end{tabular}

Boldface type indicates statistical significance $(p<0.05)$.

* Data were analyzed in 1-year increments.

vide strong, recent evidence upon which to further base these efforts.

Given their first-hand experience in managing the spectrum of ATV- and dirt bike-related brain injuries, neurosurgeons are in a unique position to advocate for increased helmet use. Our analysis provides clear and consistent summative findings for use in advocacy efforts. For example, during a 10 -year period, less than $0.5 \%$ of helmeted riders required any sort of neurosurgical intervention or died of a neurological injury. Neurosurgeons must work with those in the public health domain to disseminate this message to areas of the country that have increased instances of ATV crashes and receive disproportionately lower rates of public health education. ${ }^{6}$ In the past, states with stricter laws regarding youth recreational vehicles have had lower rates of pediatric injury and mortality.,40,41 For example, legislation passed in 2010 in Massachusetts required supervision of child riders, expanded helmet requirements, and mandated training classes for teen riders. Since implementation of this law, Massachusetts has observed sustained decreases in the rates of ED visits and inpatient hospitalizations, including a 50\% reduction in ED visits among 10 - to 13 -year-old patients. ${ }^{40}$

Further advocacy and educational initiatives are needed to continue expanding safe practices for recreational vehicle use and decrease morbidity and mortality. Messaging is critical to improving helmet use, and our analysis was purposefully constructed to support public health and injury prevention initiatives. For example, providing the unadjusted absolute risk reduction as the more tan- gible number-needed-to-helmet allows for counseling of youth and parents in the following way: "If 4 families each ensure their child wears a helmet over 10 years of ATV riding, they will collectively have prevented 1 brain bleed or fractured skull." Injury prevention programs, such as Injury Free Coalition for Kids, Safe Kids Worldwide, and ThinkFirst by the National Injury Prevention Foundation, can draw on the findings of this analysis when targeting their messaging efforts toward reducing preventable head injuries. Media campaign efforts should focus on straightforward messages from patients with ATV-related injuries, with a realistic portrayal of the possible health consequences of ATV use for young people..$^{42}$ Educational and action-oriented safety messages that inform young people about their responsibilities to learn and implement safety behaviors, as well as to educate others, are key. ${ }^{43}$

Other findings from our cohort suggest additional efforts that could be used to reduce head injury among ATV and dirt bike riders. For example, although $44 \%$ of drivers wore helmets, only $10 \%$ of passengers were helmeted. Similarly, a greater proportion of unhelmeted riders were female or were passengers, similar to the findings of other analyses..$^{14}$ Further analyses should elucidate factors associated with good riding behaviors in order to target public health interventions most effectively. Given that many ATVs and dirt bikes are designed as single-occupant vehicles, special attention should be paid to the unhelmeted passenger population presented here and in other studies. If passengers are more likely to ride unsecured or in a more precarious position, they may be at greater risk for ejec- 
tion, which was associated with increased odds of head injury in this study. In line with this reasoning, McBride et al. found that position on a vehicle may predict head and spinal injuries independent of helmet status, although this analysis was limited by its small study population. ${ }^{7}$ Other studies have supported the role of good riding behaviors in preventing injuries. ${ }^{14,18}$ Therefore, educational initiatives that focus specifically on the proper use of single-occupant vehicles, as well as helmet use, may more effectively reach this population and reduce injuries. Injury prevention and public health professionals need to focus on communitybased, multipronged prevention programs that can effectively increase knowledge and change safety behaviors. Successful campaigns should develop community coalitions and multiagency collaborations to guide community outreach, use local surveillance data, tailor inventions, and coordinate new and existing local partnerships. ${ }^{44}$

\section{Limitations}

This retrospective study had limited ability to control for the effects of confounding medical conditions and treatment decisions. We recognize the complex nature of patient care and that the patients in this study received individualized care on the basis of the judgment of the treating physicians and information available at the time. Although many of these decisions were and are protocolized (e.g., admission for all patients with acute intracranial hemorrhage), it is possible that these decisions affected some outcome variables, such as the decision to perform a neurosurgical procedure (e.g., in the event of a nonsurvivable head injury). However, close review of the health records of the 2 helmeted patients who died found that 1 patient received the single neurosurgical procedure in the helmeted group and the second patient was pronounced dead in the ED because of cardiopulmonary collapse. Additionally, owing to the nature of this study, we were unable to control for variability in the reporting of crash details, such as mechanism, location, driver status, and proper helmet size and use. Regarding helmet fit specifically, we expect that improper fit or size would only contribute to worse outcomes among helmeted patients. ${ }^{29,30}$ We also recognize that neurosurgical consultation is an imperfect proxy for injury severity but serves to describe the impact of helmet use on healthcare resource utilization and specifically neurosurgical services. Similarly, we were unable to control for the degree of other (extracranial) injuries. We expect that this may have had a confounding effect on outcome measures, such as length of hospital stay, which could have increased because of any range of injuries or other factors.

The numbers needed to helmet reported in this study should be considered estimates, because they were not adjusted for differences in demographic characteristics and injury profiles between the helmeted and unhelmeted groups. Conversely, these estimates did not include injuries that were prevented among helmeted riders who were never evaluated at a level I trauma center or by any medical professional at all; therefore, these numbers may underestimate the true effectiveness of helmets.

Lastly, this study was limited because it was conducted at a single institution serving a substantial rural popula- tion in the southeastern United States. The role and use of helmets in preventing injury likely vary according to the socioeconomic status of the study population, geographic region, and proximity to a healthcare system. These results are most applicable for informing public health interventions specific to the study region; however, these results should motivate further regional and national studies.

\section{Conclusions}

ATV and dirt bike crashes are frequently associated with head injury. In this single-institution analysis of a decade of these crashes, wearing a helmet was independently associated with decreased odds of neurosurgical evaluation, intracranial injury, and MSTBI. Most importantly, only 1 helmeted patient required a neurosurgical intervention over a 10-year period, and none required surgery such as a craniotomy or craniectomy. Despite these benefits, only one-third of those children who presented after an ATV or dirt bike crash had been wearing a helmet. There is enormous room for improvement, and neurosurgeons must work collaboratively with public health officials, injury prevention professionals, and community partners to lead these efforts.

\section{Acknowledgments}

REDCap, which was used for data management, is supported by a grant from the National Center for Advancing Translational Sciences/National Institutes of Health (no. UL1 TR000445).

\section{References}

1. Helmkamp JC, Aitken ME, Graham J, Campbell CR. Statespecific ATV-related fatality rates: an update in the new millennium. Public Health Rep. 2012;127(4):364-374.

2. Streeter RA. 2007 Annual Report of ATV-Related Deaths and Injuries. U.S. Consumer Product Safety Commission; 2009. Accessed July 27, 2021. https://www.cpsc.gov/s3fspublic/pdfs/2007AnnualATVReport.pdf

3. American Academy of Orthopaedic Surgeons, American Association of Orthopaedic Surgeons. Position Statement: All-Terrain Vehicles. 2015. Accessed July 27, 2021. https:// www.aaos.org/contentassets/1cd7f41417ec4dd4b5c4c4853218 3b96/1101---all-terrain-vehicles.pdf

4. American Academy of Pediatrics Committee on Injury and Poison Prevention. All-terrain vehicle injury prevention: two-, three-, and four-wheeled unlicensed motor vehicles. Pediatrics. 2000;105(6):1352-1354.

5. Mazotas I, Toal M, Borrup K, Saleheen H, Hester AL, Copeland D, et al. A prospective, multi-institutional study of pediatric all-terrain vehicle crashes. J Trauma Acute Care Surg. 2014;77(1):103-108.

6. Unni P, Morrow SEL, Shultz BL. Analysis of pediatric allterrain vehicle trauma data in Middle Tennessee: implications for injury prevention. J Trauma Acute Care Surg. 2012; 73(4)(suppl 3):S277-S280.

7. McBride AS, Cline DM, Neiberg RH, Westmoreland KD. Pediatric all-terrain vehicle injuries: does legislation make a dent? Pediatr Emerg Care. 2011;27(2):97-101.

8. Topping J. 2020 Report of Deaths and Injuries Involving Off-Highway Vehicles with More than Two Wheels. U.S. Consumer Product Safety Commission. 2020. Accessed July 27, 2021. https://origin.prod.cpsc.gov/s3fs-public/2020_ Report_of_Deaths_and_Injuries_Invovling_Off_Highway Vehicles.pdf 
9. Shults RA, West BA, Rudd RA, Helmkamp JC. All-terrain vehicle-related nonfatal injuries among young riders in the United States, 2001-2010. Pediatrics. 2013;132(2):282-289.

10. Helmkamp J, Lawrence BA. The economic burden of allterrain vehicle-related pediatric deaths in the United States. Pediatrics. 2007;119(1):223-225.

11. Sawyer JR, Beebe M, Creek AT, Yantis M, Kelly DM, Warner WC Jr. Age-related patterns of spine injury in children involved in all-terrain vehicle accidents. J Pediatr Orthop. 2012;32(5):435-439.

12. Lister DG, Carl J III, Morgan JH III, Denning DA, Valentovic M, Trent B, Beaver BL. Pediatric all-terrain vehicle trauma: a 5-year statewide experience. J Pediatr Surg. 1998; 33(7):1081-1083.

13. Pavlov V, Thompson-Leduc P, Zimmer L, Wen J, Shea J, Beyhaghi $\mathrm{H}$, et al. Mild traumatic brain injury in the United States: demographics, brain imaging procedures, health-care utilization and costs. Brain Inj. 2019;33(9):1151-1157.

14. Jennissen CA, Harland KK, Wetjen K, Peck J, Hoogerwerf P, Denning GM. A school-based study of adolescent all-terrain vehicle exposure, safety behaviors, and crash experience. Ann Fam Med. 2014;12(4):310-316.

15. Rodgers GB, Adler P. Risk factors for all-terrain vehicle injuries: a national case-control study. Am J Epidemiol. 2001; 153(11):1112-1118

16. Kluemper C, Rogers A, Fallat M, Bernard AC. Genitourinary injuries in pediatric all-terrain vehicle trauma-a mechanistic relationship? Urology. 2010;75(5):1162-1164.

17. Kellum E, Creek A, Dawkins R, Bernard M, Sawyer JR. Agerelated patterns of injury in children involved in all-terrain vehicle accidents. J Pediatr Orthop. 2008;28(8):854-858.

18. Denning GM, Harland KK, Ellis DG, Jennissen CA. More fatal all-terrain vehicle crashes occur on the roadway than off: increased risk-taking characterises roadway fatalities. Inj Prev. 2013;19(4):250-256.

19. Li D, Jatana KR, Kistamgari S, Smith GA. Nonfatal allterrain vehicle-related head and neck injuries to children treated in US emergency departments. Clin Pediatr (Phila). 2020;59(13):1141-1149.

20. Gorski TF, Gorski YC, McLeod G, Suh D, Cordero R, Essien $\mathrm{F}$, et al. Patterns of injury and outcomes associated with motocross accidents. Am Surg. 2003;69(10):895-898.

21. Larson AN, McIntosh AL. The epidemiology of injury in ATV and motocross sports. Med Sport Sci. 2012;58:158-172.

22. Pomerantz WJ, Gittelman MA, Smith GA. No license required: severe pediatric motorbike-related injuries in Ohio. Pediatrics. 2005;115(3):704-709.

23. Mangus RS, Simons CJ, Jacobson LE, Streib EW, Gomez GA. Current helmet and protective equipment usage among previously injured ATV and motorcycle riders. Inj Prev. 2004;10(1):56-58.

24. Carr AM, Bailes JE, Helmkamp JC, Rosen CL, Miele VJ. Neurological injury and death in all-terrain vehicle crashes in West Virginia: a 10-year retrospective review. Neurosurgery. 2004;54(4):861-867.

25. Mangano FT, Menendez JA, Smyth MD, Leonard JR, Narayan $P$, Park TS. Pediatric neurosurgical injuries associated with all-terrain vehicle accidents: a 10-year experience at St. Louis Children's Hospital. J Neurosurg. 2006;105(1)(suppl): 2-5.

26. Gibbs L, Lawrence D, Reilley B. ATV-related central nervous system injuries in Louisiana. J La State Med Soc. 1997; 149(8):276-278.

27. Hargarten SW. All-terrain vehicle mortality in Wisconsin: a case study in injury control. Am J Emerg Med. 1991;9(2): 149-152.

28. Bowman SM, Aitken ME, Helmkamp JC, Maham SA, Graham CJ. Impact of helmets on injuries to riders of all-terrain vehicles. Inj Prev. 2009;15(1):3-7.
29. Yu WY, Chen CY, Chiu WT, Lin MR. Effectiveness of different types of motorcycle helmets and effects of their improper use on head injuries. Int J Epidemiol. 2011;40(3): 794-803.

30. Rivara FP, Astley SJ, Clarren SK, Thompson DC, Thompson RS. Fit of bicycle safety helmets and risk of head injuries in children. Inj Prev. 1999;5(3):194-197.

31. Harris PA, Taylor R, Thielke R, Payne J, Gonzalez N, Conde JG. Research electronic data capture (REDCap) - a metadata-driven methodology and workflow process for providing translational research informatics support. J Biomed Inform. 2009;42(2):377-381.

32. Jennett B, Bond M. Assessment of outcome after severe brain damage. Lancet. 1975;1(7905):480-484.

33. DeCuypere M, Muhlbauer MS, Boop FA, Klimo P Jr. Pediatric intracranial gunshot wounds: the Memphis experience. $J$ Neurosurg Pediatr. 2016;17(5):595-601.

34. Peduzzi P, Concato J, Kemper E, Holford TR, Feinstein AR. A simulation study of the number of events per variable in logistic regression analysis. J Clin Epidemiol. 1996;49(12): 1373-1379.

35. Harrell FE Jr, Lee KL, Mark DB. Multivariable prognostic models: issues in developing models, evaluating assumptions and adequacy, and measuring and reducing errors. Stat Med. 1996;15(4):361-387.

36. Ganti L, Bodhit AN, Daneshvar Y, Patel PS, Pulvino C, Hatchitt $\mathrm{K}$, et al. Impact of helmet use in traumatic brain injuries associated with recreational vehicles. Adv Prev Med. 2013;2013:450195.

37. Brandenburg MA, Archer P, Mallonee S. All-terrain vehiclerelated central nervous system injuries in Oklahoma. J Okla State Med Assoc. 2005;98(5):194-199.

38. Scholten AC, Haagsma JA, Andriessen TMJC, Vos PE, Steyerberg EW, van Beeck EF, et al. Health-related quality of life after mild, moderate and severe traumatic brain injury: patterns and predictors of suboptimal functioning during the first year after injury. Injury. 2015;46(4):616-624.

39. Masel BE, DeWitt DS. Traumatic brain injury: a disease process, not an event. J Neurotrauma. 2010;27(8):1529-1540.

40. Flaherty MR, Raybould T, Kelleher CM, Seethala R, Lee J, Kaafarani HMA, Masiakos PT. Age legislation and offroad vehicle injuries in children. Pediatrics. 2017;140(4): e20171164.

41. Rattan R, Joseph DK, Dente CJ, Klein EN, Kimbrough MK, Nguyen J, et al. Prevention of all-terrain vehicle injuries: a systematic review from The Eastern Association for the Surgery of Trauma. J Trauma Acute Care Surg. 2018;84(6): 1017-1026.

42. Aitken ME, Graham CJ, Killingsworth JB, Mullins SH, Parnell DN, Dick RM. All-terrain vehicle injury in children: strategies for prevention. Inj Prev. 2004;10(5):303-307.

43. Brann M, Mullins SH, Miller BK, Eoff S, Graham J, Aitken ME. Making the message meaningful: a qualitative assessment of media promoting all-terrain vehicle safety. Inj Prev. 2012;18(4):234-239.

44. Towner E, Dowswell T. Community-based childhood injury prevention interventions: what works? Health Promot Int. 2002;17(3):273-284.

\section{Disclosures}

Dr. Yengo-Kahn serves on the scientific advisory board of BlinkTBI, which had no relevant role in study planning, execution, or funding.

\section{Author Contributions}

Conception and design: Yengo-Kahn, Allen, Vittetoe, Bonfield. Acquisition of data: Yengo-Kahn, Allen, Vittetoe, Greeno, Owais Abdul Ghani, Unni. Analysis and interpretation of data: 
Yengo-Kahn, Allen, Unni, Lovvorn, Bonfield. Drafting the article: Yengo-Kahn, Allen. Critically revising the article: Yengo-Kahn, Vittetoe, Greeno, Owais Abdul Ghani, Unni, Lovvorn, Bonfield. Reviewed submitted version of manuscript: all authors. Approved the final version of the manuscript on behalf of all authors:

Yengo-Kahn. Statistical analysis: Allen. Administrative/technical/ material support: Greeno, Owais Abdul Ghani, Lovvorn,

Bonfield. Study supervision: Yengo-Kahn, Lovvorn, Bonfield.

\section{Supplemental Information}

\section{Previous Presentations}

This work was presented virtually in abstract form at the Vanderbilt University Graduate Medical Education Research Forum, April 2021, and was presented virtually at the 2021 American Association of Neurological Surgeons Annual Scientific Meeting, August 21-25, 2021.

\section{Correspondence}

Aaron M. Yengo-Kahn: Vanderbilt University Medical Center, Nashville, TN. a.yengo@vumc.org. 